\title{
DIAGNOSTICS OF HYBRID WATER/ARGON THERMAL PLASMA JET WITH WATER, ETHANOL AND THEIR MIXTURE INJECTION TO PLASMA
}

\author{
Hlina M..$^{a, b, *}$, Maslani A. ${ }^{a}$, Medricky J. ${ }^{a}$, Kotlan J. ${ }^{a}$, Musalek R..$^{a}$, \\ HRABOVSKY M. ${ }^{a}$ \\ ${ }^{a}$ Institute of Plasma Physics AS CR, v.v.i, Za Slovankou 1782/3, CZ-182 00 Prague 8, Czech Republic \\ ${ }^{b}$ Charles Univ Prague, Dept Analyt Chem, Albertov 6, CZ-12843 Prague 2, Czech Republic \\ * hlina@ipp.cas.cz
}

\begin{abstract}
A plasma torch with the water/argon stabilization of an arc is characterized by extremely hot and high velocity plasma together with the relatively low flow rate of plasma, therefore, the torch might be properly used for plasma suspension spraying. Enthalpy probe and emission spectroscopy measurements were carried out at constant arc power with the combination of the injection of water, ethanol or their mixture to the plasma jet to diagnose the changes in temperature, heat flux and other characteristics.
\end{abstract}

Keywords: plasma spraying, suspension, enthalpy probe, spectroscopy, air entrainment.

\section{Introduction}

Suspension plasma spraying has opened new technological opportunities in the last decade [1, especially the possibility of the dispersion of very small particles in suspensions promises the interesting coating characteristics [2], [3]. Nevertheless, suspension spraying has high energetic demand due to the need of the vaporation of a solvent. The hybridly stabilized torch features extremely high-enthalpy plasma with adjustable power [4, [5], so its utilization for suspension spraying is greatly convenient (the torch has been successfully used for commercial solid state spraying in Japan and in the Czech Republic).

The injection of liquids (suspensions) to a plasma jet influences plasma characteristics and this paper shows the basic research of the changes of plasma properties measured by means of an enthalpy probe and emission spectroscopy.

\section{Experimental details}

Thermal plasma is generated in the torch with the hybrid water/argon stabilization. The argon flow is supplied along the water cooled tungsten cathode tip and is injected tangentially to assure the proper stabilization of the arc in the cathode nozzle. From the cathode region the argon plasma flows to the water vortex stabilized part, where it passes through the water channel. The length of the argon stabilized arc column is about $6 \mathrm{~mm}$, while the length of the arc column stabilized by water vortex is $50 \mathrm{~mm}$ (see Fig. 1). The diameter of the cylindrical exit nozzle is $5.7 \mathrm{~mm}$. The anode of the torch is represented by a water cooled rotating copper disc located outside of the arc chamber downstream the exit nozzle.

Plasma is formed by argon, oxygen and hydrogen ions and atoms. The mass flow rate of plasma is quite

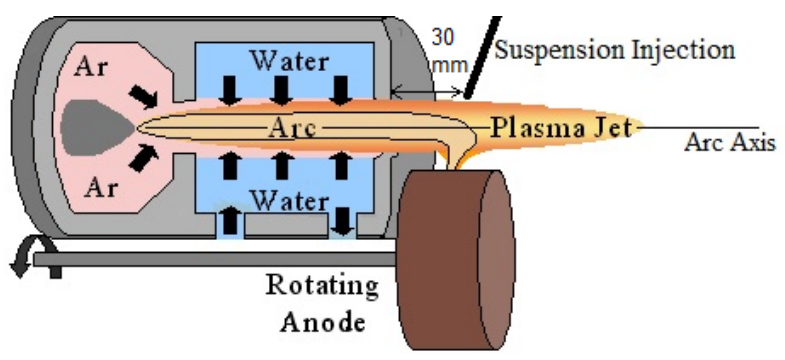

Figure 1. Schema of the plasma torch, sapphire tube for suspension injection is shown.

low $(0.3 \mathrm{~g} / \mathrm{s}$ is generated from water and approximately the same amount from argon), while exiting velocity and temperature are very high (approximately $5,000 \mathrm{~m} / \mathrm{s}$ and $20,000 \mathrm{~K}$ (centreline temperature) respectively) [4], [5]. The torch parameters were set up to $500 \mathrm{~A}(280 \mathrm{~V})$ and 12 slm (standard litre per minute) of argon to the cathode part of the torch. Liquids were injected using a sapphire tube with inner diameter of $0.35 \mathrm{~mm}$, the distance between the exit torch nozzle and the sapphire tube tip was $30 \mathrm{~mm}$ and the flow rates of liquids were set up to $95 \mathrm{ml} / \mathrm{min}$ for all cases when injection was carried out.

\section{Enthalpy probe measurement results}

The measurements were carried out by the enthalpy probe system ENP-04 produced by Tekna Plasma Systems Inc. Sampled gas is flown through a freezing unit to avoid water condensation in the system. The analysis of the sampled gas and calorific measurement enable to calculate values of plasma enthalpy, temperature, density, dynamic pressure, velocity and heat fluxes [6]. The maximum heat flux to the probe is $1000 \mathrm{~W}$, so the closest measurement axial distance 


\begin{tabular}{lll}
\hline \hline & Enthalpy [kJ/kg] & Temp. $[\mathbf{K}]$ \\
\hline 0 & 1301 & 1285 \\
$\mathrm{H}_{2} \mathrm{O}$ & 1067 & 1073 \\
$\mathrm{H}_{2} \mathrm{O} / \mathrm{EtOH}$ & 1648 & 1538 \\
$\mathrm{EtOH}$ & 2498 & 2239 \\
\hline \hline
\end{tabular}

Table 1. Enthalpy probe measurement of plasma enthalpy and temperature at axial distance of $400 \mathrm{~mm}$ for the jet without injection $(=0)$ and the 3 types of liquid injection (water $\left(=\mathrm{H}_{2} \mathrm{O}\right)$, ethanol $(50 \%$ vol.) + water $\left(50 \%\right.$ vol.) $\left(=\mathrm{EtOH} / \mathrm{H}_{2} \mathrm{O}\right)$ and ethanol $\left.(=\mathrm{EtOH})\right)$.

from the torch exit nozzle to the probe tip for all experiment cases was adjusted to $400 \mathrm{~mm}$ for set up parameters.

Table 1 summarizes the measurements of enthalpy and temperature of the plasma without injection (marked as 0 ) and the 3 types of liquid injection (water, ethanol (50\% vol.) + water (50\% vol.) and ethanol). Surprisingly, the highest values were measured in the case of ethanol injection. This phenomenon is the result of exotermic oxidation of ethanol in regions with relatively low temperature where air entrainment takes place. The same effect increases the measured values also in the case when the mixture of ethanol and water was injected. Water injection decreases enthalpy and temperature values due to the high energetic demand of heating and vaporation of the liquid. Density, dynamic pressure and velocity measurements did not reveal significant differences for the different injection types.

Heat flux to the enthalpy probe was measured as a vertical profile (axial distance $400 \mathrm{~mm}$ ) and correlates with the measurement of enthalpy and temperature - the highest values were obtained for ethanol injection, the lowest for water injection (Fig. 2). The vertical shift of the maximum value of heat flux under the axis of the torch is visible in the cases of injection (due to the momentum of injected material), while the shift above the axis occurs when no injection takes place. The shift above the axis is caused by the magnetic forces of the anode attachment of the arc.

\section{Emission spectroscopy measurement results}

Optical emission spectroscopy measurements were performed at the distance of $100 \mathrm{~mm}$ from the nozzle of the torch; this is the usual suspension spray distance for this experimental system. The overview spectra were measured by monochromator Jobin Yvon - Spex Triax 552 and iCCD detector with $1024 \times 256$ pixels. The spectral resolution was $0.16 \mathrm{~nm} / \mathrm{pixel}$ and the spectral range from $200 \mathrm{~nm}$ to $900 \mathrm{~nm}$. The arrangement of the experiment is such that at distance $100 \mathrm{~mm}$ the radiation around the jet axis with the radial range about $22 \mathrm{~mm}$ is collected and integrated. Resulting spectra are shown in Fig. 3. We compare here the plasma jet without any addition with the jet where water, ethanol and their mixture are supplied.

The common characteristic of these spectra is that in each case the dominant line is hydrogen $\mathrm{H}$ alpha at $656.3 \mathrm{~nm}$ and also lines $\mathrm{H}$ beta $(486.1 \mathrm{~nm})$ and $\mathrm{H}$ gamma $(434.0 \mathrm{~nm})$ from Balmer series together with lines of atomic oxygen and band of $\mathrm{OH}$ radical are clearly visible. It means that only species originating from water, which is one of the plasma forming media, are always detected. On the other hand, lines of argon which is also plasma forming gas are not available at this distance.

Each of the presented spectra has also its own specific features:

1. Plasma jet without injection contains lines of atomic nitrogen as well as bands of $\mathrm{NH}$ and $\mathrm{CN}$. It means that in this case the surrounding air entrains the plasma jet with the highest efficiency. Indeed, it is known that water/argon plasma jet has low density and air entrainment is very strong. It seems that injection of suspension suppresses this effect.

2. Injection of water decreases emission from all species; according expectations, water probably causes strong cooling of the plasma jet.

3. Injection of ethanol causes appearance of Swan band of $\mathrm{C}_{2}$; however, band of $\mathrm{CN}$ at $388.4 \mathrm{~nm}$ has comparable intensity as for the case without injection. It may mean that higher amount of carbon is compensated by weaker air entrainment. Another interesting feature is presence of prominent $\mathrm{Na} D$ doublet at $589 \mathrm{~nm}$, which is probably the result of small impurity in injected ethanol.

4. The case with $50 \%$ of ethanol and water injection is more similar to pure water than to pure ethanol injection, as the bands of $\mathrm{C}_{2}$ and $\mathrm{CN}$ almost disappear.

Spectra of hydrogen lines $\mathrm{H}_{\alpha}, \mathrm{H}_{\beta}$ and $\mathrm{H}_{\gamma}$ in the same distance $(100 \mathrm{~mm})$ were measured also with higher spectral resolution $(0.036 \mathrm{~nm} / \mathrm{pixel})$ in order to obtain the temperature by means of Boltzmann plot method. The cases without injection and with injection of pure water and pure ethanol were compared. Obtained temperatures were $7580 \mathrm{~K}$ for the jet without injection, $6340 \mathrm{~K}$ for the jet with injection of ethanol and $5230 \mathrm{~K}$ for the jet with injection of water. Due to different reasons (only three spectral lines; line of sight as well as radial integration of radiation; accuracy of transition probabilities; possible self-absorption), the uncertainties of these temperatures can be up to $20 \%$. However, we have still clear indication that temperature decreases with both water and ethanol injection.

\section{Discussion and conclusions}

The distance between the point of enthalpy probe and emission spectroscopy measurement differed (400 


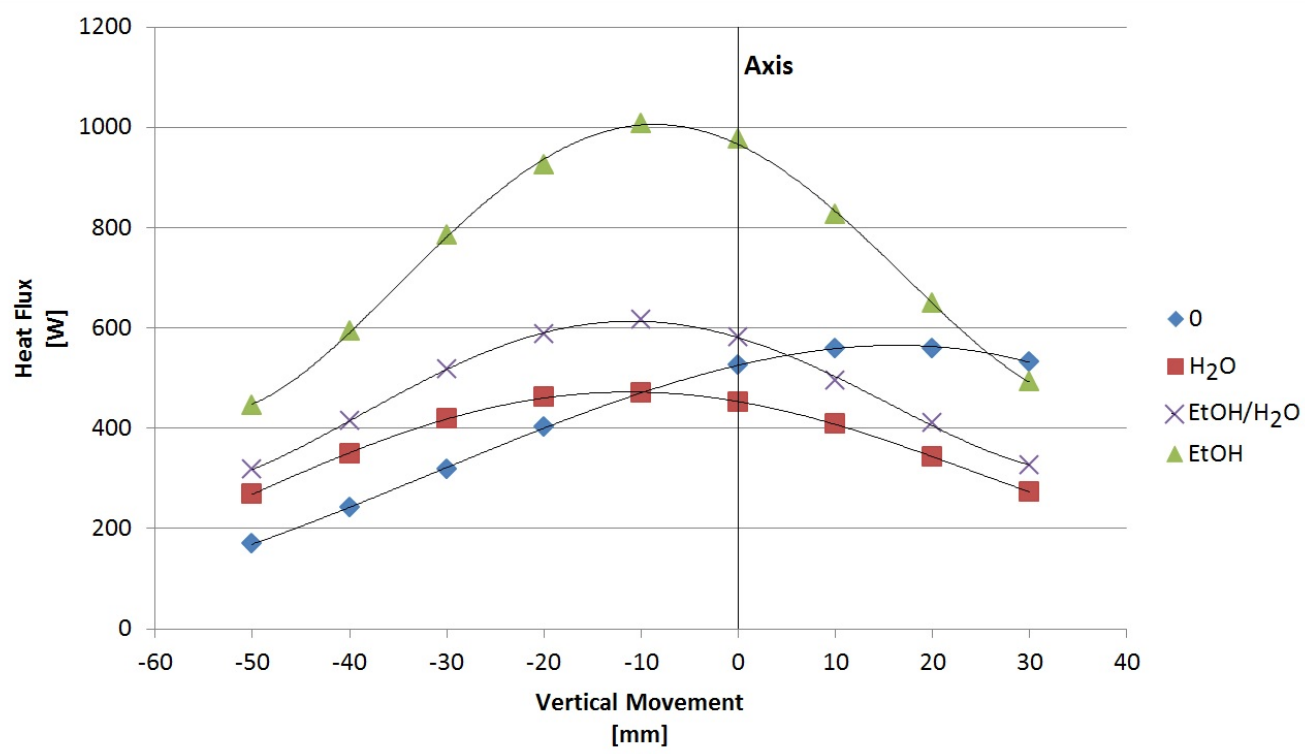

Figure 2. Heat flux to the enthalpy probe at distance of $400 \mathrm{~mm}$. Injection of water $\left(=\mathrm{H}_{2} \mathrm{O}\right)$, ethanol (50\% vol.) + water (50\% vol.) (=EtOH $\left./ \mathrm{H}_{2} \mathrm{O}\right)$, ethanol $(=\mathrm{EtOH})$ ) and without injection $(=0)$.

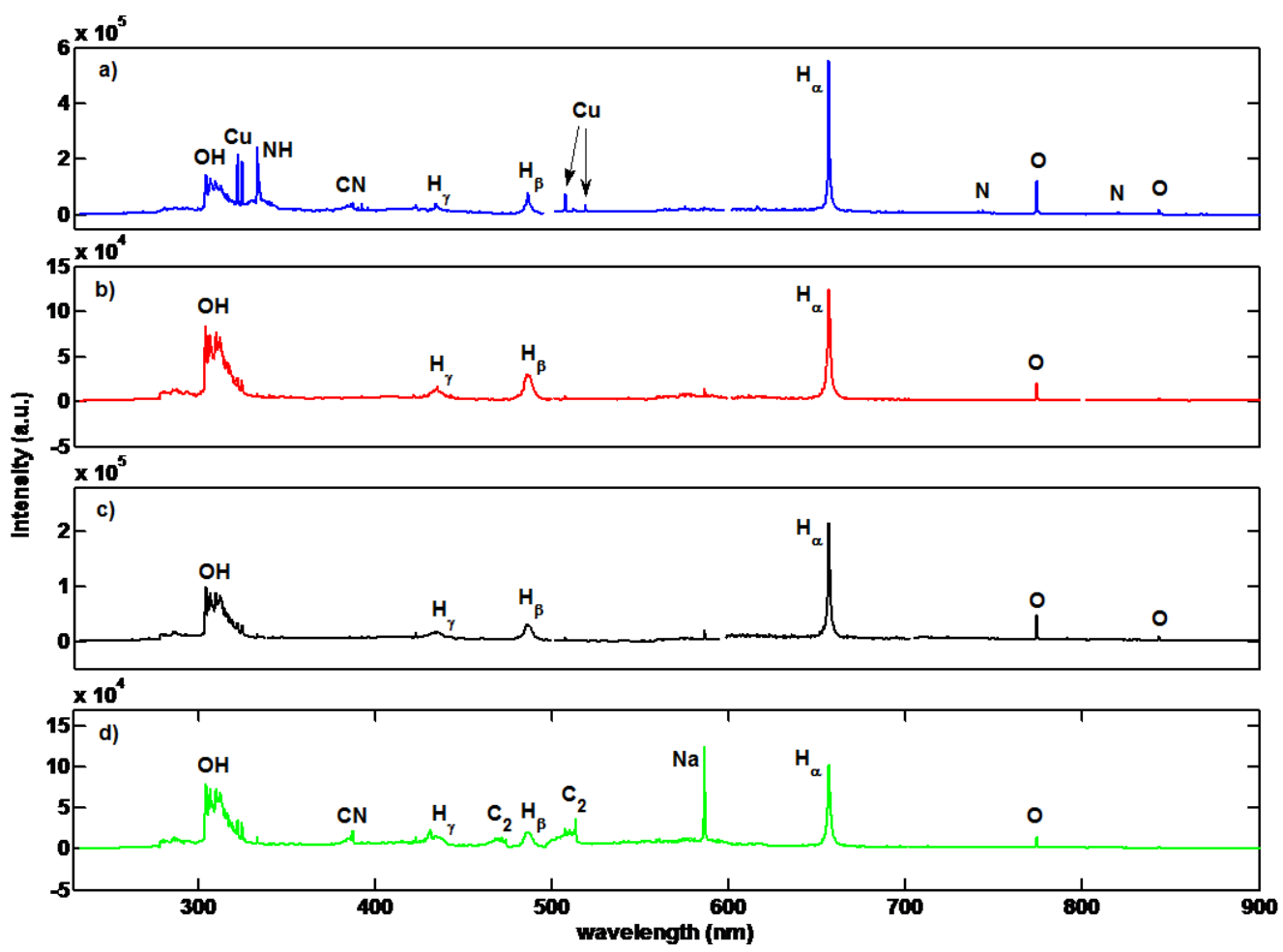

Figure 3. Emission spectra at axial distance $100 \mathrm{~mm}$ : a) no injection, b) injection of water, c) injection of mixture of water (50\%) and ethanol (50\%), d) injection of ethanol. 
and $100 \mathrm{~mm}$ ), unfortunately, but revealed dissimilarity in results can be easily explained by a gradual air entrainment to a plasma flow. The oxidation of ethanol takes place in regions with lower temperature. Such an influence can be seen at measuring distance of $400 \mathrm{~mm}$. Stronger cooling effects of water can be also explained by much higher heat of vaporization of water in comparison with the heat of vaporization of ethanol.

\section{Acknowledgements}

This research has been supported by the GA15-12145S and SVV260317.

\section{References}

[1] Fauchais P., Vardelle M., Goutier S., Vardelle A., Key Challenges and Opportunities in Suspension and Solution Plasma Spraying, Plasma Chem. Plasma P. 35, 511-525, 2014.

[2] Kotlan J., Pala Z., Musalek R., Ctibor P., On reactive suspension plasma spraying of calcium titanate, Ceram. Int.l 42, 4607-4615, 2016.

[3] Fauchais P., Rat V., Coudert J., Etchart-Salas R., Montavon G., Operating parameters for suspension and solution plasma - spray coatings, Surf. Coat. Technol. (18 ), 4309-4317, 2008.

[4] Hrabovsky M., Konrad M., Kopecky V., Sember V., Properties of water stabilized plasma torches, Thermal Plasma Torches and Technologies, vol.1, 242-266, 2000.

[5] Brezina V., Hrabovsky M., Konrad M., Kopecky V., Sember V., New plasma spraying torch with combined gas-liquid stabilization of arc, Proceedings of the 15th international symposium on plasma chemistry, vol. III, Orleans, 242-266, 2001.

[6] Hlina, M., Hrabovsky, M., Enthalpy Probe Diagnostics of Steam/Argon Plasma Jet, Plasma Physics and Technology 2, 142-145, 2015. 